\title{
Où le mythe garantit le genre : Dionysos et La Dame de Monsoreau
}

\author{
Roxane Petit-Rasselle \\ West Chester University of Pennsylvania
}

Succédant à La Reine Margot, La Dame de Monsoreau (18451846) constitue le deuxième volet de la trilogie Renaissance qu'Alexandre Dumas rédigea en collaboration avec Auguste Maquet. Henri III a remplacé son frère Charles IX à la tête d'un royaume toujours divisé par des luttes intestines. Parmi ses fidèles sujets, il compte son fou, Chicot, et ses Mignons. Il se défie de la famille des Guise et de son propre frère, le perfide duc d'Anjou, qui s'est attaché les services de Bussy d'Amboise. Blessé dans une embûche, celui-ci est soigné par la belle Diane de Méridor, dont il tombe éperdument amoureux. Hélas, elle est promise au machiavélique Comte de Monsoreau et le duc 
d'Anjou la convoite. Les deux amants, qui tentent de déjouer les plans de l'un et de l'autre, subiront leur funeste vengeance. Parallèlement, Chicot découvre la conspiration des Guise, qu'il fait échouer, sauvant son roi et son royaume. Considéré comme une œuvre "para-littéraire », Monsoreau transgresse toutes les règles du genre: alors que le roman populaire repose sur la figure du héros, d'ascendance mythique, ainsi que sur la reconnaissance, l'identification et la satisfaction du lectorat, Monsoreau raconte deux intrigues distinctes dont il est impossible de dégager un héros. En outre, il ne répond pas aux attentes qu'il a créées et provoque une frustration typique des romans dits "problématiques», non de masse. Captivant ses lecteurs alors qu'elle brise les conventions, cette œuvre peutelle être qualifiée de populaire? Après avoir présenté le roman populaire dans ses grandes lignes théoriques, puis les déviances génériques du texte dumasien, cette étude montrera comment le déplacement du mythe aux niveaux discursifs et narratifs assure la collaboration du public et garantit le genre populaire.

\section{Survol du roman populaire}

La recherche des dernières décennies a bien montré la difficulté à cerner le roman populaire en tant que genre, que ce soit par rapport au roman « élitaire », « problématique », ou bien par les mécanismes internes du texte, sa réception, l'identité de son public. Le roman populaire est souvent associé au feuilleton, puisque c'est d'abord sous ce format qu'il parut dans les quotidiens du XIX ${ }^{\text {e }}$ siècle, dont il devait assurer la vente en captivant leurs lecteurs. Pourtant, comme le rappelle Lise 
Dumasy-Queffelec, le roman-feuilleton dominait le marché de l'époque et constituait un moyen de se faire connaître. Ainsi, Balzac, Zola, Huysmans et Barbey d'Aurevilly, dont les œuvres dites problématiques parurent dans les journaux. Le roman populaire est donc plus qu'un roman-feuilleton et pourtant, il n'est pas un genre à part entière. Daniel Compère a bien noté qu'il reste avant tout « une sorte de nébuleuse à l'intérieur de laquelle il existe des genres. De plus, certains d'entre eux sont faciles à repérer et à définir (roman policier, récit historique, d'aventures, sentimental, de cape et d'épée, et de sciencefiction), d'autres sont moins aisés à cerner (roman de mœurs, récit dramatique, mystères urbains)» (p. 94). D’autres, enfin, correspondent à plusieurs genres à la fois, comme Monsoreau, qui est un roman historique, de cape et d'épée, et d'aventures.

Idéalement, le roman populaire possède, dans sa protéiformité, une formule, des mécanismes qui s'éloignent des œuvres problématiques et dont l'objectif consiste en une réception collective où la compréhension, la fascination, le plaisir, l'élan, l'assiduité et l'oubli de l'acte de lecture restent communs, en dépit des codes (politiques, sociaux, culturels, etc.) inhérents aux communautés d'accueil. Pour arriver à ses fins, le texte populaire construit la collaboration de ses lecteurs autour de trois axes: la reconnaissance, l'identification et la satisfaction. Celles-ci participent à une esthétique de la répétition, dont dépend assurément la jouissance de lecture. Ainsi sont reproduits, quoique toujours renouvelés, d'une œuvre à une autre, les mêmes héros, les mêmes systèmes de personnages, les mêmes situations, les mêmes valeurs et le même type d'action. 
(1) La reconnaissance, immanente au "para-littéraire », inclut les topoï, les "scénarios » et le héros. Parsemant le récit, les lieux communs font avancer le lectorat en terrain familier sans jamais l'exposer à d'autres réalités: ils le mettent en confiance. Il peut s'agir d'idées reçues (comme la femme madone ou diabolique), ou d'allusions mythiques vidées de leur contenu ethno-religieux et syncrétisées au niveau discursif (avec des références au diable, aux figures mythologiques, etc.). C'est donc à partir des topoï, qui puisent leur force illusoire soit dans la doxa, soit dans le mythe, que le texte fait rêver son public. La reconnaissance advient encore par ce qu'Umberto Eco qualifie de "scénarios intertextuels », c'est-à-dire qu'elle dépend des pré-connaissances livresques de son public : au fil de ses lectures, celui-ci se familiarise avec des types de scénarios comme, par exemple, les séquences d'actions (séduction, capture, torture) et même certains décors, comme les châteaux, etc. (1985, p. 103). La reconnaissance d'une action (comme la séduction) entraîne l'anticipation des étapes à venir (la capture et la torture), créant une tension et en même temps une attente : que tout rentre dans l'ordre. Non seulement, elle dirige le processus de lecture, mais elle garantit encore le plaisir de lire parce qu'elle est conforme à la taxis des autres romans populaires. Enfin, la reconnaissance, cette fois-ci du héros, est rendue possible par son essence et sa geste mythiques, sur lesquelles nous reviendrons, et par le contexte binaire (le Bien contre le Mal, la Vertu contre le Vice, etc.), qui permet à la fois de distinguer le Gentil des Méchants et de dégager les fonctions actancielles.

(2) L'identification. La reconnaissance aboutit à l'identification du lectorat. Ainsi, l'affrontement du méchant et du héros, définis par le contexte binaire, incite les lecteurs à se 
ranger dans le camp du protagoniste pour adhérer pleinement à ses valeurs. Parfois rebelle, ou bien resplendissant, beau, ténébreux, justicier ou vengeur, toujours obstiné, le héros possède une qualité essentielle qui deviendra sa "marque de fabrique » (Frigerio, 2008, p. 103) : c'est un brave, une fine lame ou un malin, bref, c'est un personnage superlatif dans un domaine particulier qui invite à l'identification, sinon par l'admiration de ses adeptes, du moins par le besoin de revanche du lecteur refoulé. En outre, le public se projette sur le personnage et sa geste grâce au procédé de la réitération. En effet, le texte exacerbe la sensibilité de ses lecteurs en leur rappelant des situations insupportables. Plus elles sont évoquées, plus le désir de résolution augmente. Le roman populaire joue de même des émotions de son public avec une structure qualifiée de «sinusoïdale» par Eco, parce qu'elle consiste en «une tension, un dénouement, une nouvelle tension, un nouveau dénouement, et ainsi de suite » (1978, p. 59). Le consommateur ne connaît aucun répit.

(3) La satisfaction. Les aventures du héros, au même titre que les réitérations narratives et discursives, produisent une tension qui exige une catharsis, tant chez le public que dans le récit. Parce qu'il répond aux attentes qu'il a créées au fil du texte et qu'il se résout toujours par une victoire du Bien sur le Mal, le récit en devient "populiste car démagogique » (Eco, 1993, p. 18). C'est bien en cela que le roman de masse apporte aux consommateurs une satisfaction qui lui est propre et qui lui permet de se distinguer du roman dit problématique, comme Le Père Goriot ou Le Rouge et le Noir, où la catharsis démêle le nœud de l'histoire, mais n'offre aux lecteurs aucune réponse satisfaisante. Tandis que le roman problématique propose différentes pistes et niveaux de lecture, le roman populaire 
dirige ses lecteurs vers une heureuse dissolution des tensions : il malmène un public qui se livre à lui avec bonheur, tombant dans ses pièges, vivant ses rebondissements, endurant mille tourments et tournant les pages, toujours haletant, toujours plus pressé, toujours plus frustré, toujours anticipant et plein d'espoir, parce qu'il n'oublie jamais la promesse de la satisfaction, déjà annoncée par les scénarios intertextuels, la couverture du livre, le titre du roman ou le nom de l'auteur.

En somme, grâce à l'alchimie de la reconnaissance, de l'identification du lectorat et de la satisfaction, la collaboration du public est source de jouissance. Et de jouissance il n'y aurait sans le héros, pilier du roman populaire. En cela, il convient de revenir sur les caractéristiques des héros contemporains de Monsoreau, puis sur leurs particularités mythiques, afin de mieux cerner le roman de Dumas. Comme le note Vittorio Frigerio, le héros populaire de la période 1830-1870 hérite des Romantiques, mais il se distingue de ceux-ci en ce qu'il s'est débarrassé de leurs incertitudes, de leur introspection et de tout respect pour l'autorité. N'admettant aucun pouvoir audessus de sa volonté, son rapport avec le monde est conflictuel, à l'inverse du héros classique, qui se montre parfaitement intégré (2008, p. 102-104). Les romans de cette époque, analyse Dumasy-Queffélec, sont «animés par l'idée que l'homme peut agir sur son destin et que la société est le champ de conflits qui font son histoire et dans lesquels l'action des hommes symbolisée par celle du héros — a un poids » (2008, p. 82). Autodidacte, indépendant et omnipotent, il dispose d'une supériorité et d'un univers idéologique qui le rendent parfaitement identifiable par rapport tant aux Méchants, qui forment l'obstacle dont il a besoin pour se construire, qu'aux 
personnages secondaires, dont il se distingue encore par son côté flamboyant et passionné.

Toujours renouvelé pour « accrocher » les lecteurs, le héros populaire reste le même d'une œuvre à une autre. Il tient d'aïeux mythiques, dont il possède certains traits, du moins les constantes retrouvées dans les romans de chevalerie et dans les épopées, ces récits qui "célèbre[nt] les premiers ancêtres mythiques ou mythifiés d'un peuple qui s'y réfère pieusement » (Kerbrat, 2000, p. 76). Le héros héroïque, rappelle Marie-Claire Kerbrat, est absent des romans réalistes, mais il survit principalement au sein des genres populaires (p. 2-3). Généralement, il est pourvu d'un charisme - « faveur divine » en grec - exceptionnel, façonné par sa grâce physique, son éclat, son orgueil et sa fierté; c'est un brave dont le sens de l'honneur traduit son souci de réputation et de gloire (p. 31-37). Or, cette gloire ne serait possible sans élan, sans une bonne part de folie et une disposition au sacrifice. De fait, le héros tend à verser son sang sans hésitation, voire sur une foucade, ce qui rend sa conduite sublime et suscite admiration et effroi. Brillant rarement par sa perspicacité et par une intelligence hors du commun, il possède une simplicité qui s'oppose à la duplicité du traître (Kerbrat, 2000, p. 67). Il n'est pas, cependant, parfaitement innocent. Il a été victime d'une injustice dont il cherche à se venger, ou encore il a été le témoin ou l'auteur d'une faute qu'il doit réparer. Cette faute originelle lui donne ainsi une mission qu'il va accomplir à la fois comme justicier et comme meurtrier. C'est un véritable parcours initiatique qui l'isole de la société et dont il revient transformé en demi-dieu. Cette ancienne structure mythique, note Dumasy-Queffelec, est souvent surdéterminée par le thème chrétien, avec la souffrance, l'expiation, la passion et la renaissance (2008, p. 76-77). 


\section{Monsoreau : De la transgression du genre à l'agression du lectorat}

Considéré comme un roman populaire, Monsoreau transgresse les règles du genre à travers la figure du héros, malmenée d'abord par l'inorganisation narrative. Racontant l'éblouissant Bussy d'Amboise comte de Clermont, le récit met soudainement en avant Chicot, le fou du roi, après dix-sept chapitres. Dès lors, le bouffon prend une importance quasiment égale à celle du premier personnage, et le héros, s'il en est un, n'est plus identifiable. La critique ne saurait s'accorder : Jacques Bonnet (p. 79) et Claudie Bernard (p.103) citent le fou, Fernande Bassan n'évoque que Bussy (p. 102) et Jacques Bony donne la préférence au duc d’Anjou (p. 7). Désignant généralement le roman non sous le nom de la jeune fille éponyme, mais sous celui de Chicot, la correspondance entre Dumas et Maquet semble traduire leur opinion: l'histrion de Henri III serait le véritable héros. Il reste que la structure du roman œuvre contre l'hypothétique volonté d'auteur de par l'espace narratif distribué équitablement entre Bussy et Chicot.

Seuls les efforts lexicaux et mythiques du texte pourraient mettre fin à l'hésitation entre les deux personnages, mais le roman choisit encore la transgression, préférant compromettre les processus de reconnaissance et d'identification du lectorat. Prenons la reconnaissance: les adjectifs attribués à Bussy témoignent de sa nette prévalence. Quatre fois, il est qualifié de "supérieur », tandis que ce même vocable n'est jamais employé à l'égard de Chicot. "Brave » décrit plus de trente fois Bussy, mais il ne se réfère que six fois au fou du roi, dont une dans le sens de «bon » et d'« honnête ». Bussy, d'ailleurs, est annoncé 
comme «le plus brave de France» ou encore «le brave des braves " - les superlatifs sont innombrables - et sa force lui vaut d'être comparé à Achille et à Roland (vol. 1, p. 79). Ainsi, l'insistance évidente du texte à l'égard du jeune homme désignerait ce dernier comme le héros du récit. D’ailleurs, à la manière de ses ancêtres mythiques, son éclat et son charisme sont reconnus de tous. En témoigne son entrée dans le roman :

Saint-Luc [...] allait répondre au roi, quand la foule, s'ouvrant, laissa voir six pages vêtus de drap d'or, couverts de colliers, et portant sur la poitrine les armoiries de leur maître, toutes chatoyantes de pierreries. Derrière eux venait un homme jeune, beau et fier, qui marchait le front haut, l'œil insolent, la lèvre dédaigneusement retroussée, et dont le simple costume de velours noir tranchait avec les riches habits de ses pages.

Bussy! disait-on, Bussy d'Amboise!

Et chacun courait au-devant du jeune homme qui causait cette rumeur, et se rangeait pour le laisser passer. (Monsoreau, vol. 1, p. 52)

Le respect qu'on lui marque et son orgueil le distinguent d'emblée des autres personnages. Du reste, sa valeur au combat et son sens de l'honneur sont célèbres, on le chante (vol. 1, p. 348), les reines le désirent (vol.1, p.182), les rois recherchent son amitié, bref, sa renommée le précède : il est une légende vivante. Ainsi, le texte impose la reconnaissance du héros par le public, mais une reconnaissance qui reste partielle. Certes, en conformité avec les héros populaires des années 1830-1870, déjà évoqués, Bussy respecte peu l'autorité et tente de rester maître de son destin. C'est aussi une fine lame qui possède toutes les qualités évoquées, un souci de gloire exacerbé, de l'élan et une part de folie, mais celles-ci se muent en faiblesse. En effet, à force de succès, Bussy «au cœur d'empereur [...] se croit digne d'une couronne » (vol. 1, p. 89) : il devient outrecuidant au point de provoquer l'ire des princes. 
Son imprudence entraîne une série de fautes qui, plutôt que de donner lieu à un parcours initiatique, mène Bussy à sa perte, prouvant qu'il n'est pas un demi-dieu et qu'il peut mourir de ses erreurs comme n'importe quel héros romanesque.

La reconnaissance du héros ne peut davantage se produire avec Chicot. Généralement décrit par sa silhouette maigre et ses longues jambes, on ne lui connaît aucun pouvoir de séduction, à l'exception de quelque amour de jeunesse qui lui vaut d'être rossé et ridiculisé (vol. 1, p. 249), de devenir victime d'une injustice dont il veut se venger. Le fou pourrait emprunter le cheminement mythique des héros populaires, avec une période initiatique dont il reviendrait transformé en personnage quasi surhumain. Mais le préjudice originel est grotesque, sa vengeance l'est encore plus et, si son adresse à l'épée est redoutable (vol. 1, p. 102 et 425), ni la voix narrative, ni les personnages n'insistent sur sa valeur au combat. Rien n'indique qu'il est invulnérable. À l'inverse de Bussy, Chicot brille par l'éloquence d'un pitre, alliée à une intelligence et une clairvoyance exceptionnelles et dissimulées, trois caractéristiques atypiques chez un héros populaire. Conscient de sa supériorité, il se déclare roi à plusieurs reprises (vol. 1, p. 512; vol. 2, p. 241), mais au lieu de s'égarer par vanité comme le fait Bussy, il n'en devient que plus calculateur. Avec sa pénétration, qui ne trouve pas sa source dans le mythique, disparaissent l'élan indispensable au héros populaire et la reconnaissance de ce dernier.

Bref, l'un est éclatant de beauté, de charisme et de gloire, pèche par orgueil et en meurt. L'autre, sans grâce, cherche à réparer une injustice, son intelligence est supérieure, ses actes calculés, et on l'associe au grotesque. Ils partagent bien 
certaines qualités héroïques, comme la constance, le sens de l'honneur et l'orgueil, qui, parce qu'elles sont communes dans la littérature, ne peuvent pallier leur insuffisance mythique. La reconnaissance partielle de Bussy et celle, impossible, de Chicot, compromettent ainsi le processus d'identification. Prenons le fou, dont on ne se soucierait plus guère si son importance narrative ne le projetait comme héros potentiel et dont la geste prévient l'admiration nécessaire à l'identification. À l'inverse du héros traditionnel, il ne verse pas son sang: il observe les autres répandre le leur (vol. 2, chapitre XCVII), car il préfère la réflexion à l'action. Sa finesse en toute situation garantit sa survie, écartant à la fois suspens et effroi des lecteurs. En outre, les paroles et les actes de Chicot l'associent au comique et au carnavalesque. Énonçant des vérités sous le masque de la folie, toujours décalé et drôle, il s'exprime à la manière des «jesters » du théâtre shakespearien que Dumas connaissait fort bien pour l'avoir adapté. D’autre part, les craintes d'un héros peuvent être dépeintes afin de prouver sa bravoure, puisqu'il parvient à les dépasser et à susciter alors l'admiration. Mais voici Chicot espionnant des conspirateurs et terrassé par une épouvante dont les manifestations le tournent en ridicule : outre son cœur battant la chamade (vol. 1, p. 274) et ses claquements de dents (vol. 1, p. 306), la sueur froide n'a de cesse de goutter sur son visage et ses mains (vol. 1, p. 306), de pointer à la racine de ses cheveux (vol. 1, p. 283), qui finissent par «se dress[er] sur sa tête" (vol. 1, p. 287); il va jusqu'à perdre l'esprit en se figurant le réveil de morts-vivants (vol.1, p. 287). Cette scène indique bien que la fonction du bouffon est moins de se prouver glorieux que de provoquer le rire dans un contexte confit de tensions. Le comique intervient encore dans la vengeance de Chicot, qui pourrait appeler un 
autre type d'identification, celle du lecteur refoulé. On se rappelle Les Trois Mousquetaires, où Aramis, bastonné comme Chicot, prend des cours d'escrime pendant un an, tire le fer contre son offenseur et lave son honneur. Le bouffon, lui, se retrouve face au cul nu de Mayenne, resté coincé dans le soupirail par lequel il cherchait à fuir (vol. 2, p. 383). Il feint d'y voir les parties charnues d'un autre, le flagelle, l'abreuve d'insultes et se nomme à diverses reprises pour s'en faire reconnaître. Loin de la rétorsion raffinée attendue depuis près de mille pages, le châtiment, qui devrait tenir lieu de catharsis, est une explosion de grotesque. Situé à la fin du récit, il confirme que Chicot n'est pas le héros, mais bien plutôt sa parodie.

Reste le vaillant Bussy, dont les qualités précédemment énoncées - l'éclat, le charisme, la beauté, la bravoure, la supériorité, la passion, l'élan - sont sources d'identification, parce qu'elles appellent d'abord la reconnaissance partielle de son essence mythique, puis admiration et effroi dans les tentatives d'assassinat, la répétition de scènes de duel, etc. Les craintes du lectorat sont renforcées par l'innocence du jeune homme, dont le cœur, resté «vierge et pur» (vol. 2, p. 13), découvre une passion qui le rend vulnérable. Véritable talon d'Achille, ses sentiments nouveaux alimentent la tension narrative et l'implication du lecteur. Pourtant, le roman, qui élabore ce fascinant héros, s'acharne à le détruire et, avec lui, l'identification qu'il avait engagée. L'assassinat de Bussy est un bon exemple de la duplicité textuelle : derrière une façade de pathos et de descriptions élogieuses, la narration mutile le jeune homme sur quatre pages avec une balle qui l'atteint à la cuisse, deux coups d'épée qui le transpercent, une dague qui s'enfonce dans sa poitrine, un couteau qui lui ouvre le jarret, 
une autre balle qui lui traverse l'épaule. Enfin, le jeune homme tombe d'une fenêtre, s'empale sur des pointes de fer, y demeure suspendu pour être achevé à bout portant. Des descriptions ultérieures ajoutent que son poing a été «coupé» (vol.2, p. 437) et « hâché » (vol. 2, p. 434). Contrairement à la tradition populaire, qui tend à abuser des références mythiques, la scène d'assassinat n'en utilise aucune, spoliant le personnage de son auréole. Malgré les quatorze hommes qu'il tue à lui seul, sa mort ressemble plus à un martyr qu'à une apothéose; il est brisé par l'ennemi. Le héros est tronqué et déchu au sens propre comme au figuré.

Le reste du récit agit pareillement, prévenant le processus d'identification par admiration en restreignant l'héroïsme du jeune comte. En effet, quasiment traqué dans ses amours avec Diane, toujours déjouant les plans de ses ennemis, il s'avère incapable, malgré sa bravoure, de résoudre des situations conflictuelles et de se dépasser. Il tente bien d'affronter les obstacles à son bonheur, mais ces derniers se retirent quand il s'en approche : le duc d'Anjou le fuit puis ourdit son assassinat, et Monsoreau le traite en ami alors qu'ils sont rivaux. Ainsi, les deux antagonistes ne sauraient jouer un rôle comparable à celui de Richelieu qui, devenu l'adversaire des trois mousquetaires, leur permet de se dégager de leur médiocrité individuelle pour se muer en héros (Petit-Rasselle, 2011, p. 986). Un héros populaire se construit face à un obstacle; Bussy, lui, est défait par l'évanescence de ses ennemis, échouant à se prouver héroïque à la manière des demi-dieux populaires.

D'ailleurs, les sept derniers chapitres lui refusent clairement ce statut, passant de la transgression du genre à l'agression du lectorat et interrogeant de ce fait tant la 
jouissance de lecture que le genre populaire de Monsoreau. D'abord, pour la raison la plus évidente : l'assassinat de Bussy, dont les traits conventionnels avaient élaboré une identification partielle, reprend la promesse de satisfaction du genre populaire et renie la collaboration du lectorat. Ensuite, avec la confirmation que Bussy n'était pas le héros et que le texte s'est joué de ses lecteurs. Le jeune homme expire sept chapitres avant la fin - depuis quand le héros meurt-il en cours de route? - et l'histoire se poursuit dans une ère post-Bussy qui n'apporte ni résolution, ni satisfaction. Ses dernières paroles étant sans portée réelle (une malédiction sans suite et le nom de sa bien-aimée), il ne laisse aucun héritage moral, sentimental, mémoriel et narratif. Ainsi, le Duc d'Anjou se voit violemment dénoncé mais reste impuni, Diane de Monsoreau disparaît abruptement et ses amis, qui cherchaient à le venger, meurent dans un carnage inutile contre les Mignons. Dans la lignée de Walter Benjamin, pour qui la mort de l'homme révèle le sens de son existence (Brooks, 1984, p. 96), la disparition de Bussy impose une lecture rétrospective : finalement, sa mort sans gloire ni retentissement est absurde - puisqu'il répand son sang pour des circonstances qu'il n'a jamais maîtrisées - et ses actions parfaitement vaines.

\section{Le mythe au secours du populaire}

L'impossibilité de dégager un héros face à l'inorganisation du récit, à la reconnaissance limitée de l'ascendance mythique des protagonistes, à la démarche textuelle brisant la seule identification possible et à la disparate de l'œuvre par rapport aux romans populaires remet en question à la fois les théories 
exposées dans notre première partie et le genre même de Monsoreau. On a vu qu'il n'est guère de roman populaire sans héros, de même que tout roman dit " paralittéraire » est censé satisfaire son lectorat, non l'agresser. Malgré ses transgressions, l'œuvre ne cesse de plaire et exerce assurément une certaine jouissance de lecture. Preuve en sont les rééditions ininterrompues de Monsoreau depuis sa première parution, la façon dont le roman releva Le Constitutionnel de la faillite lors de sa publication ainsi que les nombreuses adaptations télévisées. Si donc cette œuvre peut être qualifiée de populaire, c'est moins par les conventions du genre que par le large public auquel elle parle à travers le temps. La pérennité de l'écrit est alors envisageable, dans la pensée de Charles Grivel (2009, p. 113), comme paramètre du genre populaire.

Les traitements du héros ne contribuant pas à l'attrait de Monsoreau, il faut emprunter d'autres pistes de recherche. Parce que la présence du mythe est remarquable à plusieurs niveaux du texte et que le mythe même possède une valeur universelle qui n'est plus à démontrer, il est légitime d'en examiner la distribution dans Monsoreau en dehors des normes établies plus haut, à savoir au-delà du héros, de son ascendance mythique et de sa geste. Comme toutes les œuvres populaires, le roman de Dumas contient beaucoup d'évocations mythiques ou mythologiques au niveau discursif, mais le mythe n'offre ici aucune réponse : les allusions renvoient généralement à toutes les divinités gréco-romaines, à Satan, à Dieu, c'est-à-dire, à tout et à rien à la fois. Il s'agit d'un syncrétisme qui témoigne d'une simple volonté mythisante du texte et qui n'a guère d'impact.

En revanche, Monsoreau, qui fut écrit dans l'urgence, en même temps que La Guerre des femmes, Monte-Cristo et Le 
Chevalier de Maison-Rouge, va dans le sens de la description de Dumasy-Queffelec : «La rapidité d'écriture est particulièrement favorable à une libération de fantasmes, une explosion d'images et de scènes qui semblent parler le langage de l'inconscient. » (1989, p. 30). Or, Monsoreau pratique ce langage et s'adresse à cet inconscient par un important réseau de références à une figure mythique immanente à son contexte de réception : celle de Bacchus. Plus connu aujourd'hui comme dieu du vin, des festins et de l'épicurisme dans sa définition moderne, cette divinité se manifeste encore sous bien d'autres formes. Comme l'a montré Nathalie Mahé dans son étude de Dionysos de ses origines à aujourd'hui, le dieu s'est graduellement dissout dans notre paysage culturel avec l'absorption de ses icônes et de ses rites par l'Église, qui préféra les réinterpréter au lieu de les prohiber. En ceci, son évolution va dans le sens de l'analyse de Marie-Catherine Huet-Brichard: "L'homme, dans une société traditionnelle, adhère à un mythe constitué qu'il ne sait pas être un mythe; l'homme, dans les sociétés modernes, se méfie des mythes dont il connaît le fonctionnement, mais il vit au milieu d'eux sans le savoir » (p.34-35). Le mythe de Dionysos nous entoure mais il n'est pas toujours identifiable, masqué par un environnement christianisé.

Notons cependant qu'après avoir reculé aux XVIIe et XVIIIe siècles, Bacchus renaît ostensiblement de ses cendres dans l'Europe contemporaine à Dumas grâce aux arts musicaux, visuels et littéraires. Ainsi, pour se limiter à quelques exemples de l'Hexagone du long siècle, les opéras produits sur les scènes parisiennes de Ferdinando Paër (1811), d'Eugène Gautier (1858) et de Louis-Auguste-Florimond Ronger, dit Hervé (1892); les tableaux de Camille Corot («Silène », 1838; «Coucher de soleil avec une lionne», 18..), ceux de Paul 
Cézanne («Les grandes baigneuses », 1894-1905) et, plus tard, d'André Derain ("La dance bachique», 1906); "La mort de Penthée », de Leconte de Lisle (1852), la "Bacchante» de Guérin (1861), la «Bacchanale » d'Hérédia (1893), etc. Hugo attribue à Pan les caractéristiques de Dionysos; Dumas père et Zévaco le font revivre dans des scènes orgiaques et, pour reprendre l'analyse de Mahé, Zola use du mythe dans La Curée et La Faute de l'abbé Mouret (p. 301-303).

Que Dionysos se manifeste de manière visible ou non, son immanence culturelle peut être évoquée légitimement ainsi que le façonnement de la disposition mentale du public. Comme toutes les divinités, idéologies et images-forces encore actives, Bacchus agit comme une convention, à savoir comme un phénomène ayant un pouvoir fédérateur auprès d'un public qui ne croit plus aux mythes mais qui reste demandeur de fascination, de vérités et d'absolus. En d'autres termes, Bacchus a perdu son pouvoir et ses valeurs ethno-religieux face à l'évolution de son public et à travers la série de décontextualisations et de re-contextualisations qu'il a subie, mais il continue de séduire. Parce qu'elle se meut au gré des aléas socio-historiques, cette convention n'est pas inaltérable et peut disparaître et, avec elle, la fascination du public. Alors qu'elle provient de la culture, de l'extérieur, la convention s'inscrit dans le texte littéraire, dont elle fixe le code. Elle s'y diffuse en sous-texte mais elle peut faire surface par l'évocation d'un nom, d'une caractéristique, etc. Aussi, dans le cadre d'un roman populaire tel que Monsoreau et à la suite de Bassan, pour qui «l'espace mythologique [...] représent[é] par le feuilleton répond à la soif de sacralisation, de divinité au sein d'une société déchristianisée » (p. 102), souhaitons-nous ajouter que c'est non le syncrétisme mythique, déjà évoqué, mais un 
ensemble mythique cohérent et courant dans son contexte d'accueil qui répond aux attentes du public. En effet, la coïncidence d'un code mythique entre le texte et son public permet à ce dernier de s'immerger facilement dans l'œuvre et de s'identifier pleinement non à son héros, comme le voudrait tout roman populaire, mais à son univers. Là, se situent la jouissance de lecture et la satisfaction du public.

Nous avons mentionné que, masqués par des valeurs chrétiennes, nombre d'invariants dionysiaques ne sont plus attribués aujourd'hui à la divinité mais qu'ils peuplent néanmoins le paysage culturel. Ainsi, l'illusion et la révélation, l'ambivalence sexuelle et la palingénésie. Comme nous allons le voir pour Monsoreau, il s'agit là de faisceaux de traits et de pans de récits mythiques par lesquels la convention dionysiaque produit un contexte binaire qui ne permet pas de prendre parti pour un personnage plutôt qu'un autre (comme le ferait, par exemple, le Bien contre le Mal), mais d'assurer les trois critères essentiels à toute œuvre populaire par le procédé de la réitération: la reconnaissance, l'identification et la satisfaction du lectorat.

Situant le roman dès sa phrase d'introduction, "Le dimanche gras de l'année 1578...» (vol.1, p.45), le texte témoigne de sa volonté mythisante et de l'orientation qu'il donnera à l'histoire. En effet, la Renaissance, où se déroule Monsoreau, assimile le carnaval à Dionysos et le célèbre selon sa tradition, et ce, à juste titre, puisque les origines onomastiques de cet événement remontent au carrus navalus, soit le char naval de Bacchus (Mahé, 1992, p. 200). Ainsi, le ton est donné dès la première ligne de Monsoreau : tout ne pourrait être que leurre. C'est la couverture prometteuse du roman populaire qui enferme une multitude de frustrations, c'est Chicot le sage qui 
se cache derrière le fou, c'est le vain Bussy sous le lustre du héros. Entamer un roman sous le signe de la divinité, c'est donc promettre une oscillation constante entre l'illusion et la révélation, selon la fonction première de Bacchus, qui fut d'abord le dieu du théâtre.

Et le texte va tenir parole, d'abord par son utilisation de l'accessoire théâtral par excellence : le masque. Le portent ceux qui enlèvent Diane de Méridor, le duc d'Anjou lors de l'assassinat de Bussy et, plus comique, Henri III, sous forme de pâtes cosmétiques. Ensuite, par l'entrée de Chicot dans le roman, Chicot qui joue de son costume pour tromper son public :

au moment où apparaissait à l'une des portes le roi Henri III, un autre Henri III, exactement pareil au premier, vêtu, chaussé, coiffé, fraisé et godronné de même, apparaissait par la porte en face. De sorte que les courtisans, un instant emportés vers le premier, s'arrêtèrent [...] et refluèrent en tourbillonnant du premier au second roi (Dumas, 1995, vol. 1, p. 49).

Le fou s'annonce comme le maître de la mascarade, puisqu'il se fait passer pour son souverain, mais surtout pour un esprit carnavalesque, qu'il conserve tout au long du roman. À l'instar des bouffons de la fête, il se cache sous le masque de la folie et du comique pour critiquer les nouveaux impôts (vol. 1, p. 242), brocarder les Mignons (vol. 1, p. 243), rudoyer et tutoyer le roi, s'en faire servir, etc. La mascarade se poursuit au niveau narratif avec la geste souterraine de Chicot, une geste qui récrit l'histoire de France et par laquelle il devient à la fois le double populaire d'Henri III et le régent insoupçonné du royaume. En somme, selon la tradition carnavalesque et toute bachique, Chicot brise la hiérarchie sociale en jouant de l'illusion et de la révélation. 
Celles-ci se poursuivent avec un autre invariant dionysiaque, celui de l'ambivalence sexuelle. Rappelons que la divinité est tantôt représentée en amphigame, tantôt dépeint en vieillard masculin et barbu ou en jeune homme frêle, imberbe et efféminé. Quelles que soient ses représentations, Bacchus est célébré pour sa virilité tout en étant associé aux femmes : c'est un dieu conquérant, guerrier et à la forte sexualité, dont la majorité des adeptes sont féminins. Sa véritable nature est insaisissable et Henri III s'en fait l'écho. Qualifié d'«hermaphrodite antique» (Dumas, 1995, vol. 1, p. 99), il s'entoure de Mignons, préfère les garçons à son épouse et s'enduit de pâtes et de crèmes afin de préserver sa beauté. Ces masques, qui se veulent indiquer le côté efféminé, faible, capricieux et ridicule de celui qui les porte, sont levés plus tard pour dévoiler masculinité, force physique, adresse aux armes et détermination. L'évolution d'Henri III ne témoigne pas d'un retour à une «norme » virile et hétérosexuelle, au contraire. Tant que le texte lui attribue une féminité exacerbée, le roi joue un rôle antagoniste. Il faut attendre que son ambivalence soit dévoilée pour qu'il se mue en un personnage positif. Son hermaphrodisme trouve alors toute sa justification et devient une norme acceptable, historique, mythique, diffusée dans le récit et reconnaissable de tous.

Parmi les autres motifs mythiques prolongeant l'illusion et la révélation, et facilement identifiables, la palingénésie. Aujourd'hui communément assimilée au Christ, elle est le propre de Dionysos, qui est associé aux enfers, à la mort et à tout acte destructeur, mais aussi à la création, à la vie et à ses plaisirs. Selon les versions, les Titans tuent, dépècent et dévorent l'enfant-dieu que Zeus ressuscite, ou bien il vient deux fois au monde, d'abord dans l'utérus de Sémélée, puis dans la 
cuisse de Zeus. Or, la palingénésie se retrouve trois fois chez Dumas. D'abord avec Diane, dont le voile, flottant sur les eaux des douves du duc d'Anjou, fait croire à la noyade. Ensuite, Bussy qui, grièvement blessé, revient à la vie grâce à Rémy Le Haudoin. Enfin, Monsoreau est vaincu à l'épée par Saint-Luc qui, le pensant perdu, prononce son oraison funèbre et annonce son décès. Voilà trois personnages déclarés morts, dont les résurrections servent de ressort à l'intrigue. Agissant au niveau narratif, elles participent à la tension et au suspens nécessaires au roman populaire, renouvellent la promesse dionysiaque de l'introduction, poursuivent ainsi la stratégie textuelle et maintiennent le lectorat en situation de reconnaissance et d'identification. Idem pour la mort de Bussy, dont le corps déchiqueté rappelle le «sparagmos», soit le démembrement dont est victime Bacchus enfant.

Alors que l'illusion et la révélation, avec l'ambivalence sexuelle et la palingénésie, sont disséminées dans le texte littéraire, elles restent les traits ou pans de récits les moins attribués à Dionysos par le grand public. En revanche, l'image d'une joyeuse divinité de la vigne, héritée des XVIe, XVIIe et XVIIIe siècles, reste la plus identifiable avec son association à la boisson et à la bonne chère, bref, à la joyeuse vie. Monsoreau évoque des omelettes, des poulardes, des bons crus et des odeurs de fricot chatouillant la narine. Il met en scène victuailles et beuveries, de grands mangeurs, des gourmands, des boulimiques ou de ridicules jeûneurs. Dionysos tronqué et réduit à sa plus simple expression, son évocation agit à la manière d'une synecdoque: les allusions, les dialogues, voire les chapitres entiers autour de la table, s'ils ne font progresser l'intrigue - de fait, ils en détournent les lecteurs - ont pour fonction de faire émerger Bacchus tout entier à la surface du 
texte et de provoquer explicitement la reconnaissance du public. D'ailleurs, c'est dans ce contexte que la divinité est nommée ouvertement (vol. 1, p. 146, 255 et 264). Le texte prolonge son effet au niveau narratif, avec, par exemple, les chapitres XVIII et XIX, où Chicot saoule Gorenflot afin de proférer à sa place une harangue qui, galvanisant les Ligueurs, donne à l'histoire un tournant tant nécessaire qu'attendu. Quel que soit le niveau textuel de sa manifestation (discursif ou narratif), la divinité ramenée à ses traits les plus matériels donne à l'ensemble de références bachiques toute sa cohérence. Il ne saurait ainsi être question de "syncrétisme mythique ", mais d'un motif mythique constant.

\section{Conclusion}

Sans la dissémination du mythe dionysiaque dans le récit, Monsoreau ne pourrait prétendre au populaire. On a vu que le texte, qui limite d'abord la reconnaissance de son héros, s'acharne à le briser, décevant les attentes de son lectorat et transgressant les règles. Or, la dissémination du mythe de Dionysos dans le texte va garantir le genre. Alors qu'il a perdu ses fonctions étiologiques, pseudo-scientifiques et ethnoreligieuses et qu'il est masqué par la culture chrétienne, Bacchus séduit encore un public toujours en demande de fascination, d'absolus et de vérités. Diffusé dans Monsoreau sous forme de faisceaux de traits ou de pans de récits, il fixe le code du roman et coïncide avec la culture d'accueil. À la manière des topoï, le mythe dionysiaque produit d'abord un terrain familier : il expose les lecteurs à des « réalités » connues 
et les met ainsi en confiance. En outre, avec l'invariant de l'illusion et de la révélation, perpétuées par l'ambivalence sexuelle et la palingénésie, il élabore un contexte dichotomique qui ne permet ni de discerner les fonctions actancielles, ni de prendre partie pour un personnage. En revanche, il perpétue la tradition binaire du roman populaire en recréant un univers idéologique qui invite à l'identification. L'atmosphère, les personnages, leurs mœurs et leur geste bachiques agissent également auprès des lecteurs. C'est donc grâce au déplacement du mythe, qui n'opère plus dans la figure du héros mais aux niveaux discursif et narratif sous les traits de Bacchus, que sont garantis les processus de reconnaissance, d'identification et de satisfaction. L'esthétique de la répétition inhérente au populaire est ainsi altérée tout en étant préservée à travers les mécanismes de lecture et la reprise du mythe. La jouissance de lecture, qui repose en grande partie sur la conformité du roman avec les autres, est assurée.

Avec le va-et-vient entre le texte littéraire et le mythe aux niveaux discursif et narratif, Monsoreau n'est pas une récriture du mythe de Bacchus ou même, dans la perspective de Claude Lévi-Strauss (1968, p. 95-105), un exemple de dégénérescence du mythe en littérature. Monsoreau n'est pas non plus un mythe littéraire. En effet, il ne peut exercer une fascination telle qu'elle entraîne des centaines de suites et de récritures à la façon des Mousquetaires: au lieu de rattacher des caractéristiques mythiques au héros, Monsoreau les diffuse dans le récit. En revanche, ce roman permet de mieux saisir la stratégie d'écriture issue du tandem Dumas-Maquet. Nombre de leurs romans enfreignent les règles du genre populaire. D'abord, avec un héros concurrencé par un autre, comme avec Chicot et Bussy, ou d'Artagnan, graduellement supplanté par Athos. Ou 
encore, leurs actions et leurs défauts sont tels que l'identification du lectorat devrait être impossible: Athos pend sa femme au coin d'un bois, Porthos est un imbécile et Coconnas, assoiffé de sang, massacre les Huguenots. Ou enfin, des romans comme Margot, les Mousquetaires et Monsoreau créent des attentes chez les lecteurs sans y répondre. Quelles que soient leurs transgressions, ils pallient une éventuelle frustration par un réseau cohérent de références au dieu de la vigne. D'autre part, la présence d'éléments bachiques dans Monsoreau permet d'envisager le travail de Dumas historien sous un nouvel éclairage. De fait, le récit reflète cette Renaissance qui engendra le théisme, qui renouvela l'orphismedionysisme et qui intégra Bacchus dans sa vision chrétienne jusqu'à le considérer comme un double du Christ (Mahé, 1992, p. 217-221) : c'est une poularde grasse que Chicot affirme être une carpe en plein Carême, c'est du vin qui sert à baptiser (vol.1, p. 264), c'est le couple Henri III-Chicot, avec le monarque qui jeûne et se flagelle par dévotion chrétienne en opposition avec son fou qui fait ripaille. Dumas est loin de raconter la dynamique originale entre le roi et son bouffon, dont le rôle historique fut restreint, mais le mélange d'éléments bachiques et chrétiens et les innombrables festins sur fond de la Ligue transmettent tout un syncrétisme religieux et mythique inhérent au Rinascimento. Le Dictionnaire de cuisine de notre auteur révèle de solides connaissances de la divinité grecque. Dumas joua-t-il sciemment d'éléments bachiques ou ne fit-il que libérer des fantasmes issus de son inconscient et de celui de son époque? 


\section{Bibliographie}

BASSAN, Fernande. (1993-1994), «Le roman-feuilleton et Alexandre Dumas père. (1802-1870)», Nineteenth-Century French Studies, vol. 22, no 1, p. 100-111.

BERNARD, Claudie. (1996), Le Passé recomposé. Le roman historique français du dix-neuvième siècle, Paris, Hachette.

BONNET, Jacques. (1978), « Où le lecteur découvre comment et pourquoi il arrive que des qualités sans héros dissimulent un héros sans qualités », L'Arc, vol. 71, p. 76-81.

BONNY, Jacques. (1995), "Introduction», dans Alexandre Dumas, La Dame de Monsoreau, Paris, Flammarion, p. 5-42.

BROOKS, Peter. (1984), Reading for the Plot. Design and Intention in the Narrative, Harvard, Harvard University Press.

BRUNEL, Pierre. (1992), Mythocritique. Théorie et parcours, Paris, Presses universitaires de France.

COMPÈRE, Daniel. (2009), "Vous avez dit roman populaire? ", dans Le Rocambole. Bulletin des amis du roman populaire, Paris, Belles Lettres, p. 93-101.

COUÉGNAS, Daniel. (2008), «Qu'est-ce que le roman populaire? », dans Loïc Artiaga (dir.), Le Roman populaire 1836-1960. Des premiers feuilletons aux adaptations télévisuelles, Paris, Éditions Autrement, p. 35-53.

DUMAS, Alexandre. ([1845-1846] 1995), La Dame de Monsoreau, Paris, Flammarion. 
—. ([1844] 1972), Les Trois Mousquetaires, Paris, Librairie générale française.

—. ([1872] 1998), Mon dictionnaire de cuisine, Paris, UGE Poche.

DUMASY-QUEFFÉLEC, Lise. (2008), «Univers et imaginaire du roman populaire», dans Loïc Artiaga (dir.), Le Roman populaire 1836-1960. Des premiers feuilletons aux adaptations télévisuelles, Paris, Éditions Autrement, p. 75-95.

ECO, Umberto. ([1978] 1993), De Superman au Surhomme, trad. Myriem Bouzaher, Paris, Grasset \& Fasquelle.

-. ([1979] 1985), Lector in fabula, trad. Myriem Bouzaher, Paris, Grasset.

FRIGERIO, Vittorio. (2008), «Bons, belles et méchants (sans oublier les autres) : le roman populaire et ses héros », dans Loïc Artiaga (dir.), Le Roman populaire 1836-1960. Des premiers feuilletons aux adaptations télévisuelles, Paris, Éditions Autrement, p. 97-116.

-. (2002), Les Fils de Monte-Cristo. Idéologie du héros de roman populaire, Limoges, Pulim.

GRIVEL, Charles (2009), "Populaire: le vrai-faux combat», dans Le Rocambole. Bulletin des amis du roman populaire, Paris, Belles Lettres, p. 113-117.

HUET-BRICHARD, Marie-Catherine. (2001), Littérature et mythe, Paris, Hachette.

KERBRAT, Marie-Claire. (2000), Leçon littéraire sur l'hérö̈sme, Paris, PUF.

LÉVI-STRAUSS, Claude. (1968), L'Origine des manières de table, Paris, Plon. 
MAHÉ, Nathalie. (1992), Le Mythe de Bacchus, Paris, Fayard.

PETIT-RASSELLE, Roxane. (2011), « Le problème du héros dans Les Trois Mousquetaires », The French Review, vol. 84, p. 978990.

QUEFFÉLEC, Lise. (1989), Le Roman-feuilleton français au XIXe siècle, Paris, PUF.

Schopp, Claude. (2002), Alexandre Dumas, Paris, Fayard.

—. (2010), Dictionnaire Alexandre Dumas, Paris, CNRS Éditions.

\section{Résumé}

Le roman populaire repose sur la figure du héros, d'ascendance mythique, ainsi que sur la reconnaissance, l'identification et la satisfaction du lectorat. La Dame de Monsoreau transgresse toutes les règles du genre : la reconnaissance, l'identification et la satisfaction sont impossibles face à l'absence d'un héros véritablement populaire. Cet article propose de repenser les paramètres du "para-littéraire», un genre ici garanti par le déplacement du mythe : ce n'est pas la nature du héros ou le syncrétisme mythique, présent à la surface du texte, mais un réseau cohérent de références à Bacchus aux niveaux narratifs et discursifs qui assure la collaboration du public.

\section{Abstract}

Popular novels are based on the hero, who is of a mythical essence, along with the process of recognition, identification and readership's satisfaction. La Dame de Monsoreau 
transgresses all the rules of this genre. The absence of a truly popular hero prevents any recognition, identification and satisfaction. By redefining the parameters of the popular novel, this article explores how the latter is supported by the displacement of the myth. It is not the nature of the hero, not the mythical syncretism at the surface of the test, but a coherent network of references to Bacchus on the narrative and discursive levels that allows the readers' collaboration. 